\title{
Active Flow Separation Control at the Outer Wing
}

\author{
J.P. Rosenblum ${ }^{(1)}$, P. Vrchota ${ }^{(2)}$, A. Prachar ${ }^{(2)}$, S.H. Peng ${ }^{(3)}$, S. Wallin ${ }^{(3)}$, P. Eliasson ${ }^{(3)}$, P. Iannelli( ${ }^{(4)}$, V. \\ Ciobaca $^{(5)}$, J. Wild ${ }^{(5)}$, J.L. Hantrais-Gervois ${ }^{(6)}$, M. Costes $^{(6)}$ \\ ${ }^{(1)}$ Dassault Aviation, (2) VZLU, ${ }^{(3)} \mathrm{FOI},{ }^{(4)} \mathrm{CIRA},{ }^{(5)}$ DLR, ${ }^{(6)}$ ONERA
}

Within the European project AFLoNext one of the technological streams is dedicated to the investigation of Active Flow Control (AFC) to increase the robustness of the wing tip design at takeoff conditions, while allowing the optimization of aerodynamic efficiency in cruise. AFC is used to delay the wing tip stall, which is caused by vortex breakdown, thus improving the lift to drag ratio and allowing a steeper climb gradient during second segment climb when the landing gear is retracted.

The numerical parametric studies of AFC aerodynamic sizing were shared between the involved partners on the basis of actuator location (in the leading edge region or on the upper surface) and actuator types (steady blowing, synthetic jets, pulsed jets). Most of the numerical results have been obtained for steady blowing through continuous or segmented slots. The specific effect of the unsteady means of actuation on the flow topology was also identified. The aim of this study was to take into account industrial requirements defined by Airbus and geometric constraints of AFC actuators arising from the project partners involved in the development of AFC hardware. Some comparisons between the partners' results are presented, allowing preliminary conclusions to be drawn. The most effective and efficient device turned out to be pulsed blowing through segmented slots located at the leading edge separation line.

\section{Nomenclature}

$\begin{array}{ll}\alpha & \text { Angle of Attack } \\ C_{D} & \text { Drag coefficient } \\ C f x & \text { Longitudinal skin friction coefficient } \\ C_{L} & \text { Lift coefficient } \\ C_{m} & \text { Pitching moment coefficient } \\ C_{\mu} & \text { Blowing momentum coefficient based on wing reference surface } \\ C p & \text { Pressure coefficient } \\ \text { L/D } & \text { Lift to drag ratio } \\ Q & \text { Second invariant of the velocity gradient tensor }\end{array}$

\section{Abbreviations}

AFC Active Flow Control

AoA Angle of Attack

CAD Computer Aided Design

CFD Computational Fluid Dynamics

IDDES Improved Delayed Detached Eddy Simulation

LE Leading Edge 


$\begin{array}{ll}\text { LES } & \text { Large Eddy Simulation } \\ \text { PJA } & \text { Pulsed Jet Actuator } \\ \text { RANS } & \text { Reynolds Average Navier Stokes } \\ \text { SJA } & \text { Synthetic Jet Actuator } \\ \text { TS } & \text { Technology Stream } \\ \text { UHBR } & \text { Ultra-High Bypass Ratio }\end{array}$

\section{Introduction}

This study is part of the AFLoNext project (Active Flow - Loads \& Noise control on Next generation wing) within the European Union $7^{\text {th }}$ Framework Program. One of the main goals is the application of Active Flow Control (AFC) techniques locally on several regions of the wing. The objective is to assess the potential benefit either for retrofit to current aircraft or for future aircraft designs. An overview of the different Technology Streams (TS) in the project is depicted in Figure 1. In TS2 the application of AFC technology is investigated on the outer wing region. A second scenario for AFC technologies is addressed in TS3, where Flow Control is applied on the wing/pylon junction to counter the lift losses due to the closely-coupled integration of Ultra High Bypass Ratio (UHBR) turbofan engines. The other Technology Streams deal with other approaches for reducing emissions for future aircraft, such as hybrid laminar flow control or noise and vibration mitigation.

The wing/pylon junction and the outer wing are the two regions of a commercial airliner wing prone to separation at low speed, high lift conditions, since they are not protected by a slat, as illustrated by Figure 2. The design of wing tip extensions is a compromise between drag minimisation in cruise and satisfactory aerodynamic behaviour at low speed, high lift conditions. Usually, wing tips or winglets are designed so that they do not stall at take-off, which may lead to a reduced performance benefit in cruise. Therefore, a strategy for improving this compromise may be to optimize the wing tip for drag minimisation in cruise and rely on Active Flow Control (AFC) to delay the wing tip stall at take-off. Thus this study was dedicated to the investigation of the potential brought by AFC.

The take-off performance is an important objective for any transport aircraft. The general design of a high-lift system must match airfield efficiency requirements in terms of take-off field length, and climb rate [1], [2]. The performance is significantly dependent on the aerodynamic efficiency of the airliner. During take-off drag and lift-to-drag ratio are largely impacting this performance. An increased aerodynamic efficiency can result in shorter ground-roll distance and/or steeper climb slope, but also in a larger number of passengers and/or reduced engine size.

Today's civil transport aircraft are designed with large and highly curved wing tip devices for minimizing drag at cruise, decreasing environmental impact and reducing fuel burn. The geometry of these wing tip devices is various, but the local sectional airfoils are typically thin and no leading edge (LE) devices, such as Krüger, slat or dropped nose are installed. The flow on the wing tips cannot withstand large angles of attack without separating. A local separation bubble forms on the upper side of these wing devices which stall much earlier than the high-lift wing, drag increases and lift-todrag decreases. Because the separation suppression on the wing tip can significantly improve the airliner aerodynamics TS2 is dedicated to research of an active new technology that could reduce or eliminate the local flow separation. The transport aircraft aerodynamics at take-off shall be enhanced by active flow control [3],[4] applied at the wing tip. Through delaying potential flow separation on 
the outer wing at take-off, AFC will help in increasing lift and reducing drag, thus improving the liftto-drag ratio (L/D) and leading to a steeper climb gradient in the second segment of climb (when the landing gear is retracted), which will potentially benefit the 'one engine operative' case.

The selected methods to control the local flow are:

- steady blowing (or constant blowing), which needs air-supply. The available massflow is limited according to engine bleed air availability.

- synthetic jet actuation (SJA), i.e. a zero-net-mass flux method combining blowing and suction phases, which offers the large potential to be applied at the outer wing region without requiring airfeeding.

- pulsed jet actuation (PJA), which requires plumbing and air supply. The pulsing of the jet is obtained by combining blowing and no blowing phases at a given frequency either with a square or sinusoidal dynamic signal.

Most of the actuator types in existence at the start of the project (especially SJA) deliver lower exit velocity peaks and momentum than required for a realistic aircraft application. Therefore during the project further improvement of the actuators was also targeted (see [5] and [6]). The research of AFC for outer wing application is so new that a quantification of the geometrical (position, size, shape, direction) and dynamic parameters (exit velocity peak, mass flow, frequency) for the actuators must be performed prior the design of large scale actuators. These aerodynamic challenges have been tackled through numerical simulations by several partners within the project.

The control of flow separation using unsteady actuation is often reported to be most effective when applied close to the separation line, where the base flow shows the largest instabilities. Research on controlling flow separation indicates that using these instabilities the flow may be controlled with a minimum amount of added energy [3], [4]. Therefore, it is of interest to place actuators close to the leading edge of the wingtip device, which has been investigated by most of partners. Previous research also suggests that actuators should also provide large velocity ratios with respect to the local flow in order to generate strong vortices to delay separation. The flow on the wing tip is vortexdominated and interacting with local vortex also becomes crucial. Applying AFC in a high-speed, vortex dominated flow, like that present at the leading edge region of the wing tip, therefore becomes less favourable due to the high actuator velocities and momentum required to have any influence on the flow.

Ciobaca and Wild [7] showed that controlling a wing tip flow separation at low speed by pulsed switching jets is feasible and that actuating close to the leading edge is more favourable than placing the actuators in a more downstream position. However, larger flow unsteadiness was computed with the translation of the slots towards the leading edge. Ciobaca et al. [8] presented results of experimental studies confirming the success of fluidic actuation for wing tip separation control, and underlined the importance of the positioning and the distribution of these actuators. On the basis of wind tunnel test results Bauer et al. [9] analysed the efficiency of the slot switching jet actuators at the wing tip and close to the leading edge and explored the impact of placing the AFC-modules at different spanwise locations on the wingtip. Stall delay by more than $2^{\circ}$ angle of attack was achieved with the minimum energy input when only the inboard actuators were active. Such a conclusion led 
Detinis et al. [10] to investigate other AFC devices such as steady suction at the root leading edge of the wing tip, which they studied numerically and experimentally on a notional outer wing configuration within the AFLoNext project.

Therefore, the objective of the present study was to investigate the potential of other AFC devices than the sweeping or switching jets to control wing tip flow separation and to analyse the influence of their location on the outer wing of an airliner.

TS1 Hybrid Laminar Flow Control on wing and fin

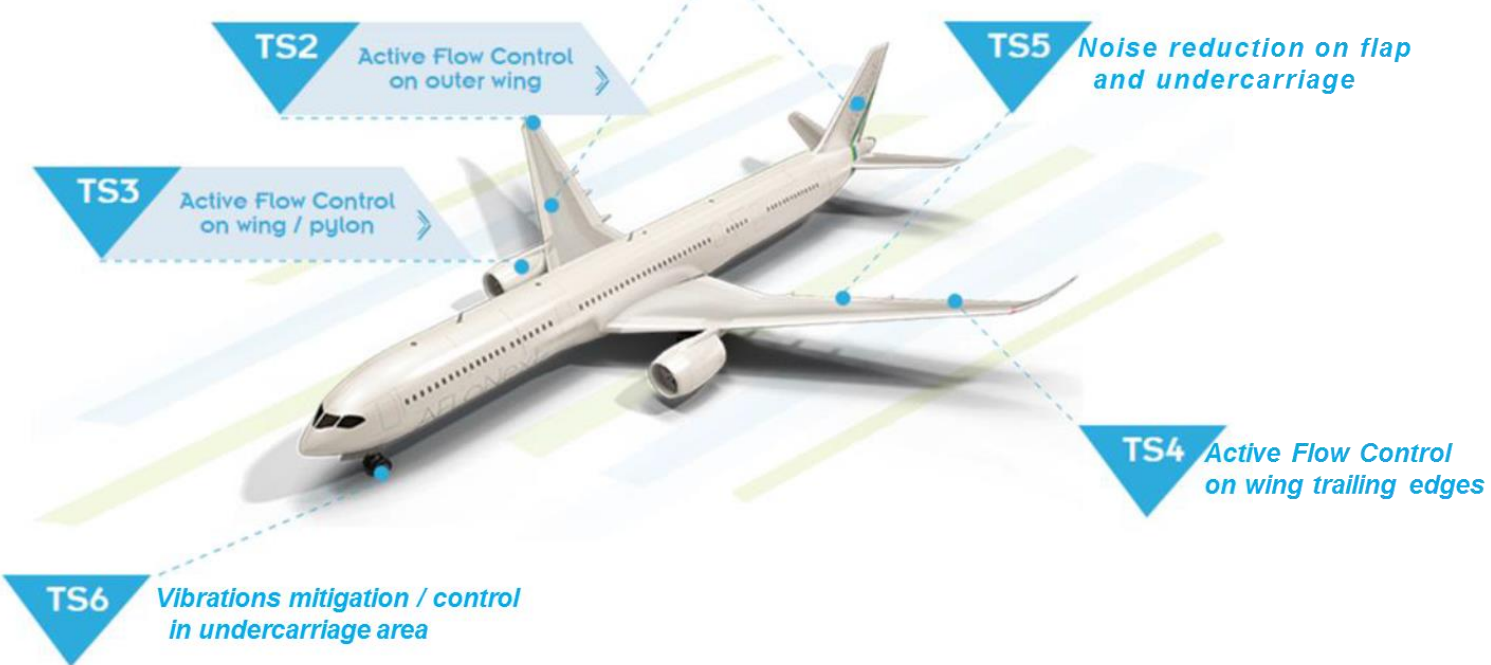

Figure 1: Overview of Technology Streams for the AFLoNext project

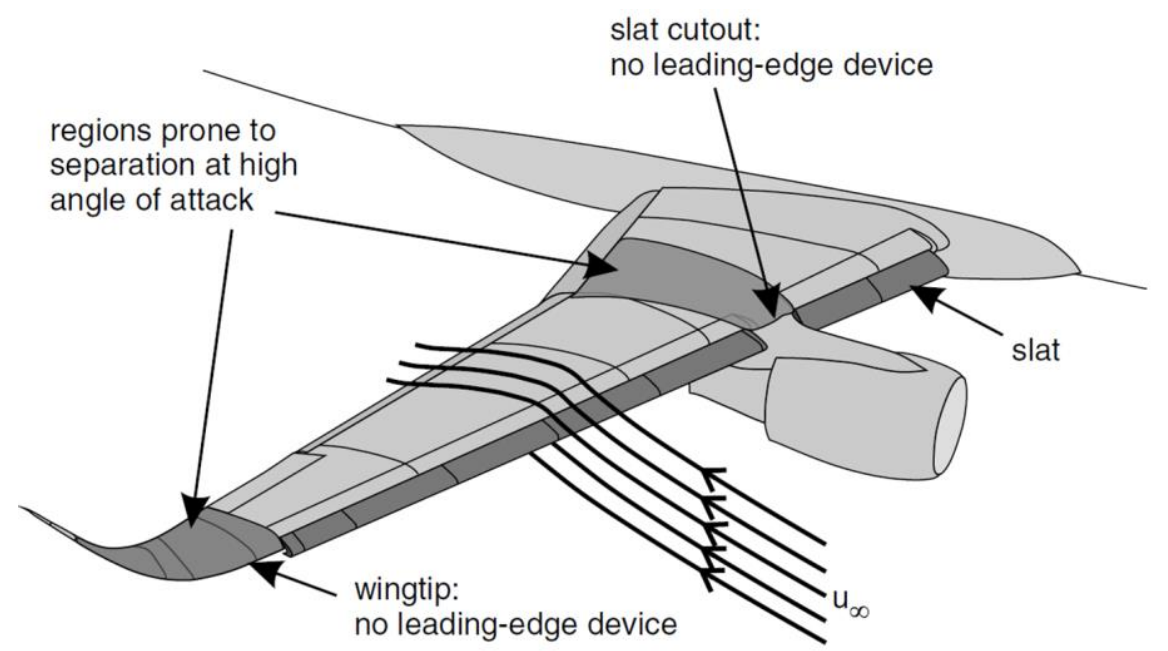

Figure 2: Representation of regions prone to separation in low speed, high lift conditions: wing/ pylon junction and wing tip (see Bauer et al. [9]) 
The CAD geometry of a research aircraft configuration named XRF1 was provided by Airbus Group to the partners for this project. Airbus designed a wing tip extension with a curved leading edge (see Figure 3) compatible with reducing drag in cruise.

In the following sections the characterisation of the baseline outer wing at take-off will be presented first. This will be followed by comparing the effect of unsteady AFC using synthetic jets to the one of steady blowing. Afterwards, taking into account a number of actuator design constraints arising from hardware development, an investigation of their influence both on steady blowing and pulsed blowing cases will be performed. Finally a synthesis will be drawn on the basis of the partners' results comparison.

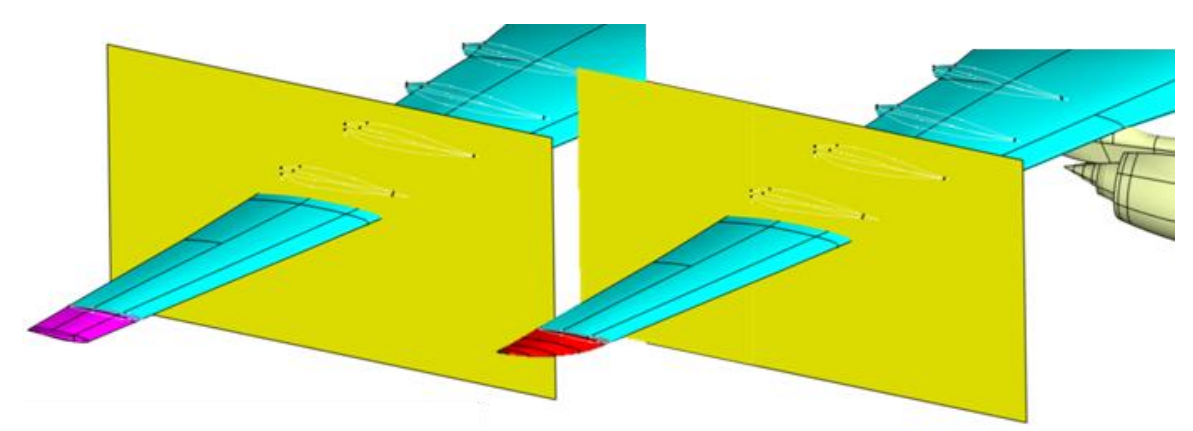

Figure 3: Configuration of wing tip devices designed for the study: on the left with straight leading edge, on the right with curved leading edge. The yellow plane corresponds to the interface plane for the simplified geometry.

\section{Baseline characterisation at take-off}

The flow about the baseline wing was characterized at both cruise and take-off conditions. Two types of geometries were considered: The full aircraft configuration and a geometry limited to the outer wing region, as defined by the interface plane shown in Figure 3 . The flow topologies and spanwise loading were found to be in good agreement between these two geometries, provided a shift of nearly $+2^{\circ}$ was taken into account on the angle of attack for the simplified geometry. The limitation of the geometry was mainly motivated by the need to perform costly unsteady computations when simulating active flow control configurations.

Aircraft level aerodynamic performance results, which were obtained by ONERA using elsA software [11], are illustrated in Figure 4, showing that wing tip stall occurs between 14 and $15^{\circ}$ of incidence. Its effects are characterized by a kink in the lift curve $C_{L}(\alpha)$, which corresponds to a slope reduction. It is also associated with a drag increase, a L/D reduction and a destabilising pitch up moment increment. The related skin friction lines evolve from the footprint of a vortex on the whole wing tip extension to a wing tip dominated by fully separated flow (see Fig. 5).

Figure 6 presents the results on the simplified geometry limited to the outer wing, which were produced by Dassault Aviation with proprietary Navier Stokes code Aether (see [12] and [13]). The reduction in lift, the increase of drag and the reduction in L/D curve occur between $16^{\circ}$ and $17^{\circ}$ of 
incidence, which is $2^{\circ}$ higher than for the full geometry. Dashed lines represent the target of improvement which is desired from AFC. The associated flow topology is characterized by Figure 7. The vortex footprint which is seen on wing tip extension is the one which is generated by the slat edge. When incidence increases, the tip vortex moves inboard and creates destabilising flow conditions which promote the breakdown of the slat end vortex.

Based on previous experience several flow control strategies were proposed taking into account the baseline flow characteristics. They can be summarized as follows:

- One of the most efficient locations for AFC is the leading edge separation line on the wing tip in order to prevent the inboard movement of the tip vortex and its destabilizing effect.

- Application of the AFC at the wing tip root where it can be used to strengthen the slat end vortex and delay its bursting.

- Integrating AFC on the upper surface may make its integration easier than in the region of the curved leading edge and may contribute to the slat end vortex reinforcement.

- Applying AFC in the leading edge region between the attachment line and the separation line might help to reduce the peak velocities required of the actuator jets.

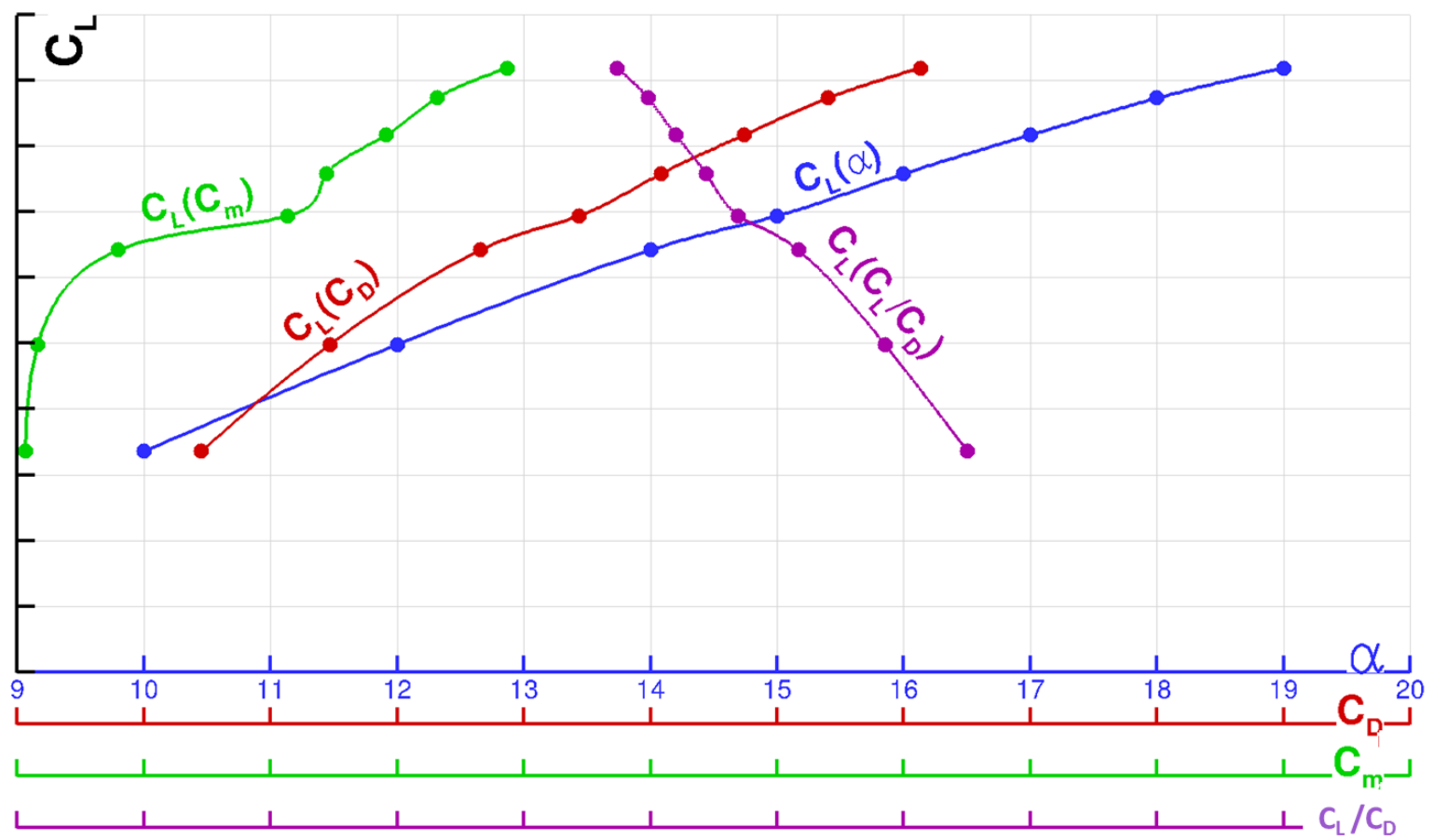

Figure 4: lift curve, drag polar, pitching moment curve and $C_{L} / C_{D}$ for the full aircraft configuration at take-off 

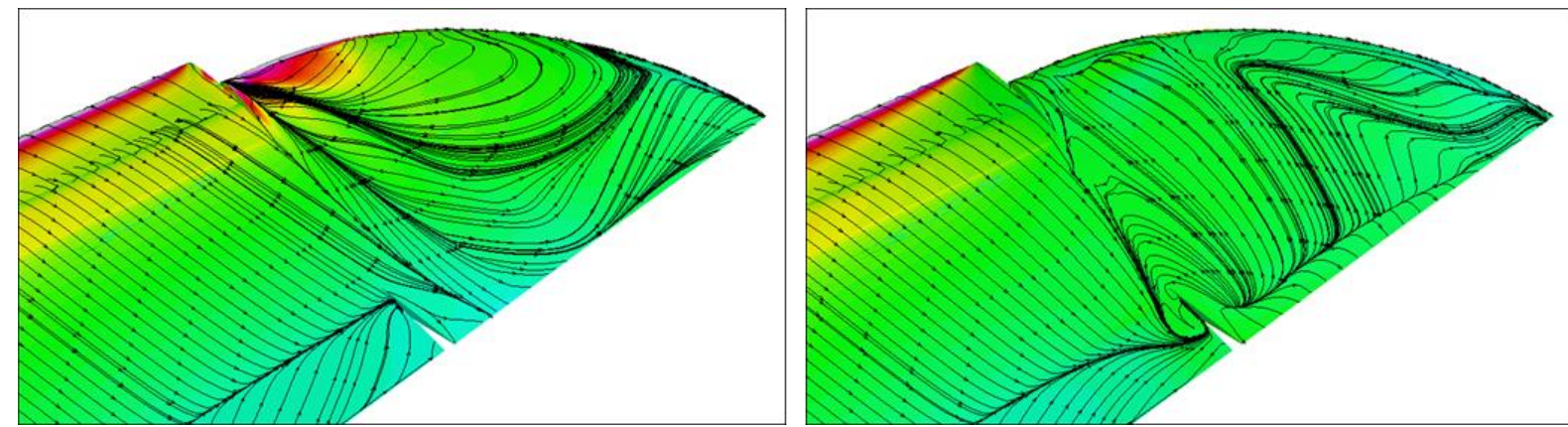

Figure 5: Pressure distribution and skin friction lines for 14 and 15 degrees angle of attack on the wing tip of the full aircraft configuration
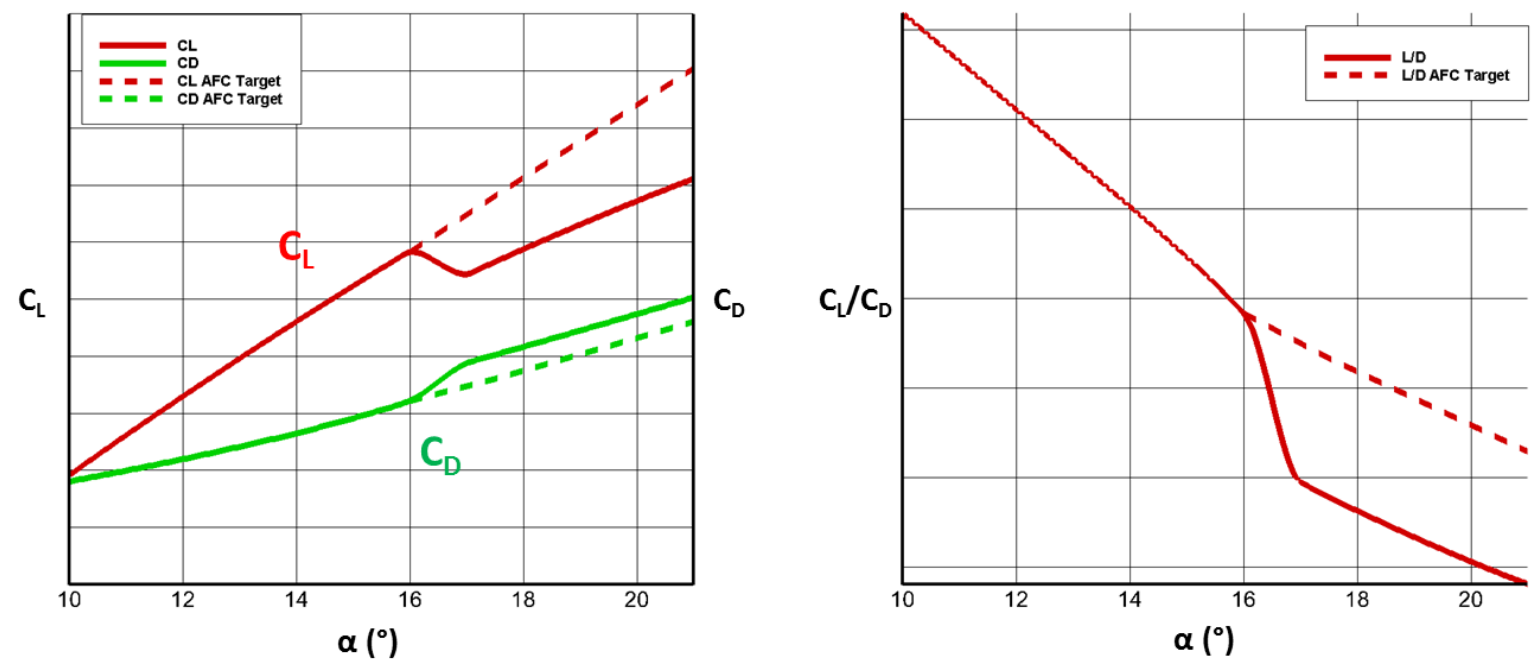

Figure 6: Lift, drag and $C_{L} / C_{D}$ curves (solid lines) on the baseline simplified geometry and target curves with AFC (dashed lines)

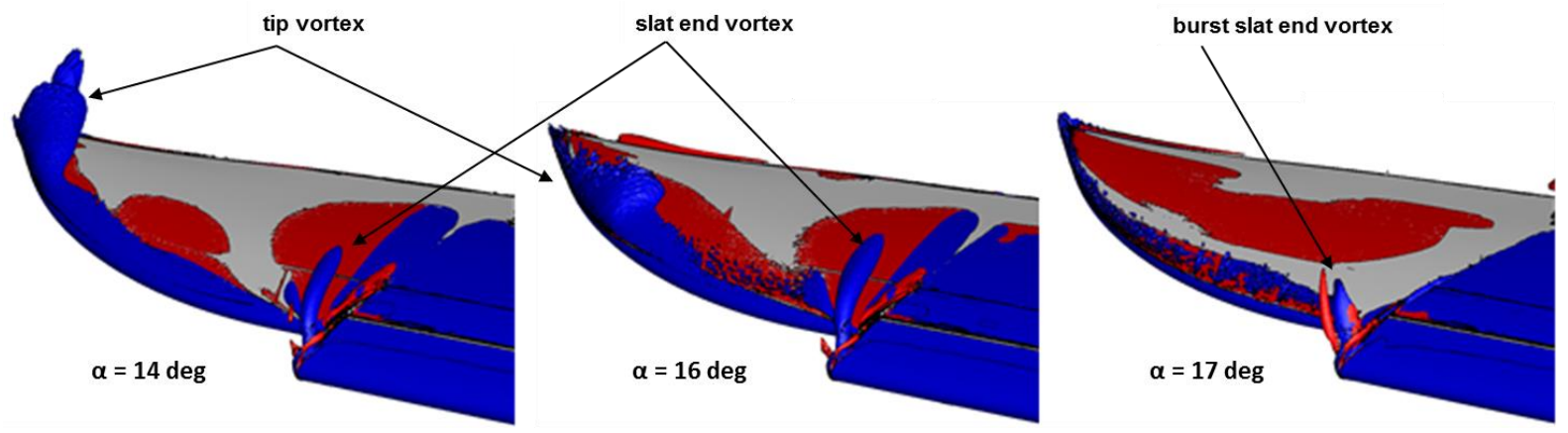

Figure 7: Flow topology on the simplified geometry deduced from $Q$ criterion $(Q=0.075)$ coloured by longitudinal vorticity (blue clockwise, red counter clockwise in front view) for $14^{\circ}, 16^{\circ}$ and $17^{\circ}$ angle of attack 


\section{Comparison of Synthetic Jets and steady blowing on the wing tip upper surface}

First AFC studies were performed with wide blowing slots in order to look for effectiveness in delaying the wing tip stall, especially for the AFC concepts located on the upper surface away from the leading edge separation line, for which an increase in the required actuator mass flow was expected. Discrete, closely spaced synthetic jet actuators with spanwise slot-like exit geometry were investigated by DLR on the wing tip upper surface located at $10 \%$ of chord (see Figure 8 ). The choice of the chordwise position of the actuators was decided taking into account the fact that the velocity ratio of the actuator jet to the incoming flow, which is considered crucial for the success of delaying flow separations, should exceed a value of 2 . Some parametric studies were firstly done on the blowing slot width with steady blowing actuation, leading DLR to select $4 \mathrm{~mm}$ wide slots to get a satisfactory effectiveness of AFC. The geometry of the actuator exit is based on 20 slots which are 4 $\mathrm{mm}$ wide, $100 \mathrm{~mm}$ long and with a pitch angle of $30^{\circ}$ to the wing tip surface.

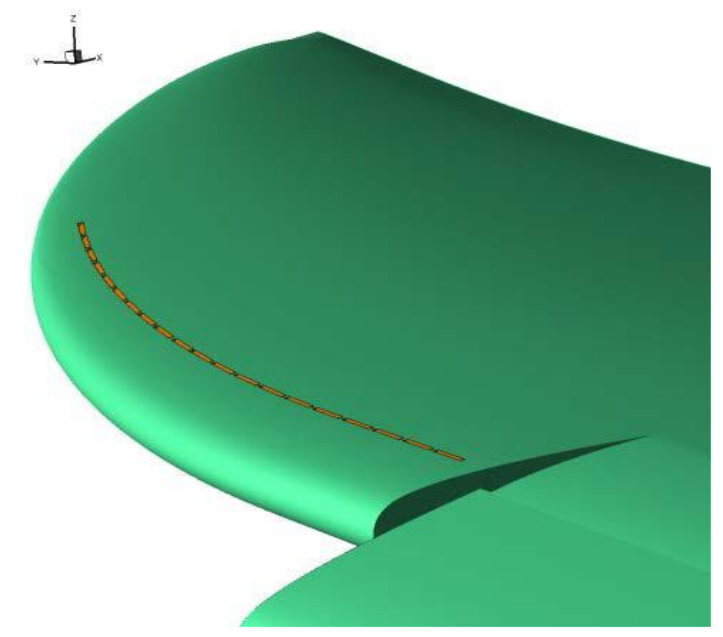

Figure 8: Location of the SJA slots on the wing tip upper surface

The RANS and URANS computations were performed using the finite volume compressible flow solver TAU developed at DLR [14] using a Spalart-Allmaras turbulence model, which represents DLR best practice for high lift configurations with or without flow separation control (see References [15], [16] and [17]).

Figure 9 presents the influence on lift and drag of the two types of actuation (continuous blowing and synthetic jet actuation). Continuous blowing at $273 \mathrm{~m} / \mathrm{s}$ (blowing at Mach 0.8) allows postponement of the wing tip stall by more than $3^{\circ}$ of incidence and results in additional lift increment, due to the blowing effect on the surface pressure. At an incidence of $21^{\circ}$ the corresponding lift is higher than the baseline. In comparison to the baseline, drag is reduced for angles of attack between 14 and 19 degrees.

For the synthetic jet actuation (SJA) two peak jet velocities were considered for investigative purposes: $273 \mathrm{~m} / \mathrm{s}$ and $180 \mathrm{~m} / \mathrm{s}$. The actuators were all in phase along the wing tip span. For SJAs, which combine blowing and suction phases, two types of signal of jet exit velocity with time were computed, either sinusoidal or square signal. The results obtained with both were very similar. Only 
results related to sinusoidal time signals are presented in Figures 9 and 10. The actuation frequency was chosen to be $100 \mathrm{~Hz}$, which is a compromise between having a large local Strouhal number and not using too high frequencies when performing URANS simulations with a dual time step method. It should be noticed that the synthetic jet actuators manufactured by the AFLoNext partner Fraunhofer have a frequency in the order of $1.5 \mathrm{kHz}$ [5]. The most appropriate approach to simulate such an actuator would be LES, which is not feasible today for the flight Reynolds number of an airliner. Figure 9 shows that the gain in lift and drag is reduced when compared to steady blowing, but the SJA is still effective in the same range of incidence. Decreasing the SJA peak velocity slightly reduces the actuator effectiveness. Similar conclusions can be drawn from the lift to drag ratio L/D in Figure 10.

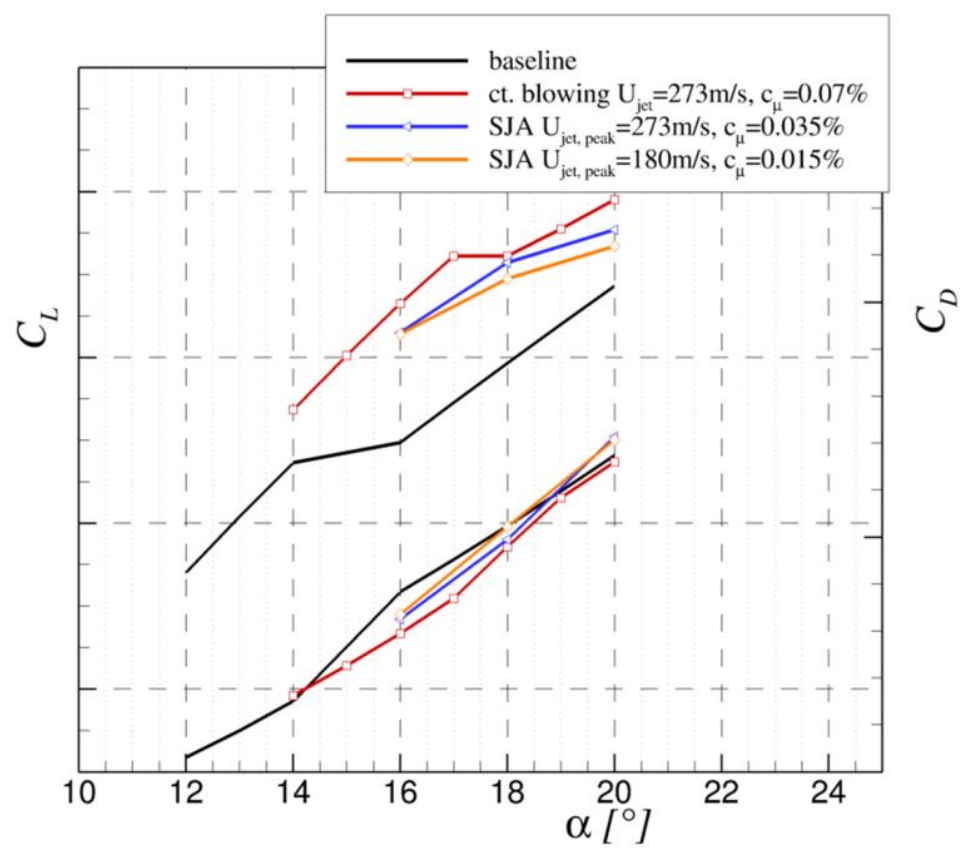

Figure 9: Lift and drag curves for the baseline, continuous blowing $\left(U_{\text {jet }}=273 \mathrm{~m} / \mathrm{s}\right.$, massflow $=1.3 \mathrm{~kg} / \mathrm{s}$, $\mathrm{C} \mu=0.07 \%)$ and synthetic jets SJA1 $\left(U_{\text {jet peak }}=273 \mathrm{~m} / \mathrm{s}, C \mu=0.035 \%\right)$ and $\mathrm{SJA2}\left(\mathrm{U}_{\text {jet peak }}=180 \mathrm{~m} / \mathrm{s}\right.$, $\mathrm{C} \mu=0.015 \%$ ) on the outer wing

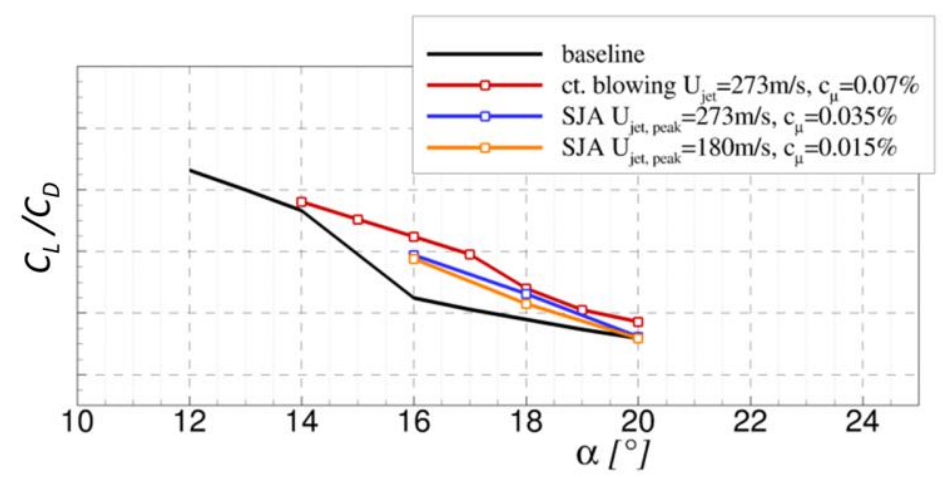

Figure 10: $C_{L} / C_{D}$ curves for the baseline, continuous blowing $\left(U_{\text {jet }}=273 \mathrm{~m} / \mathrm{s}\right.$, massflow $=1.3 \mathrm{~kg} / \mathrm{s}$, $\mathrm{C} \mu=0.07 \%)$ and synthetic jets SJA1 $\left(U_{\text {jet peak }}=273 \mathrm{~m} / \mathrm{s}, C \mu=0.035 \%\right)$ and $\mathrm{SJA} 2\left(\mathrm{U}_{\text {jet peak }}=180 \mathrm{~m} / \mathrm{s}\right.$, $\mathrm{C} \mu=0.015 \%$ ) on the outer wing 
The analysis of longitudinal skin friction coefficient $\mathrm{Cfx}$ and skin friction lines at $18^{\circ} \mathrm{AoA}$ in Figure 11 helps understanding of the different flow mechanisms involved. At this incidence the flow is fully separated on the baseline wing tip. The flow is partially reattached for the continuous blowing case, due to massive momentum injection in the boundary layer, which redirects the flow in the direction normal to the slots and pushes the slat end vortex footprint towards the trailing edge. The skin friction lines are completely different for the two synthetic jet cases, for which the wing tip upper surface is dominated by the footprint of the slat end vortex, which is reinforced in the wing tip root region by a local increase of $\mathrm{Cfx}$. The synthetic jet actuation generates rows of spanwise vortices which travel in the streamwise direction and help stabilize the flow and strengthen the slat end vortex (fig. 12).
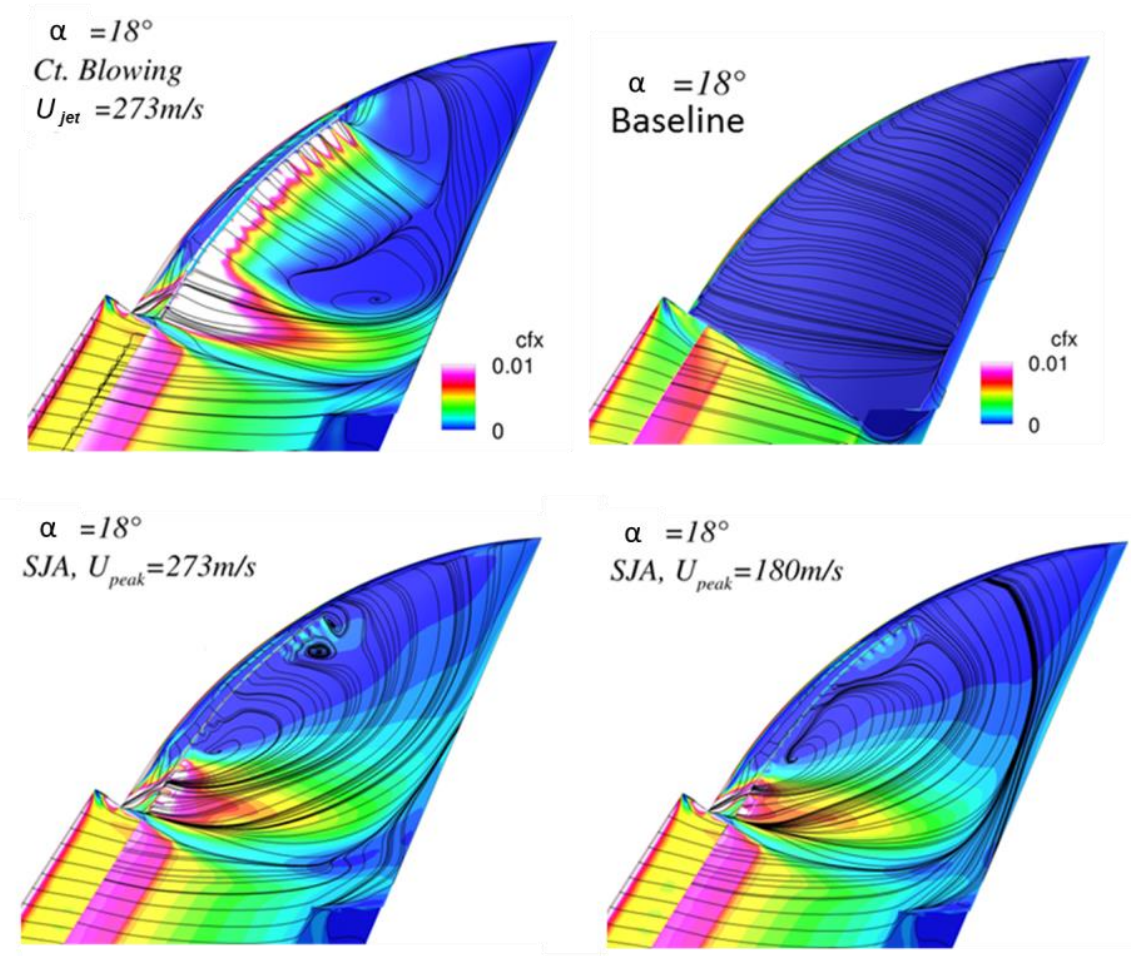

Figure 11: $\mathrm{Cfx}$ and skin friction lines at $18^{\circ}$ AoA for the baseline, continuous blowing $\left(\mathrm{U}_{\mathrm{jet}}=273 \mathrm{~m} / \mathrm{s}\right.$, massflow $=1.3 \mathrm{~kg} / \mathrm{s}, \mathrm{C} \mu=0.07 \%)$ and synthetic jets SJA1 $\left(\mathrm{U}_{\text {jet peak }}=273 \mathrm{~m} / \mathrm{s}, \mathrm{C} \mu=0.035 \%\right)$ and SJA2 $\left(U_{\text {jet peak }}=180 \mathrm{~m} / \mathrm{s}, \mathrm{C} \mu=0.015 \%\right)$ on the outer wing

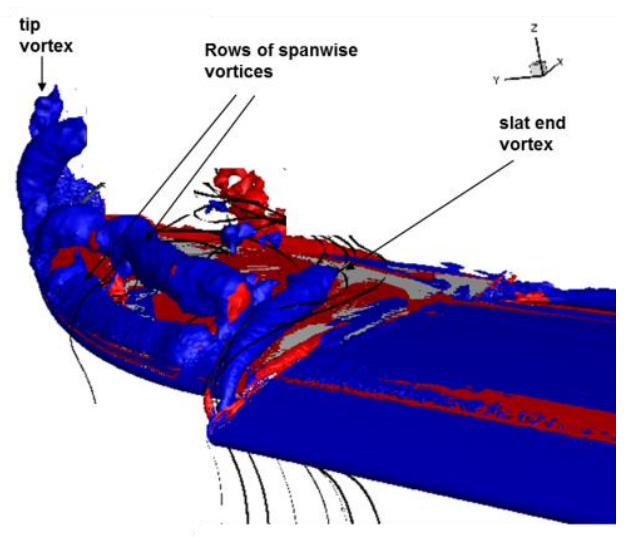

Figure 12: Instantaneous flow topology for the synthetic jet actuation $\left(U_{\text {jet peak }}=273 \mathrm{~m} / \mathrm{s}, \mathrm{C} \mu=0.035 \%\right)$ at $18^{\circ}$ AoA based on $Q$-iso surfaces colored by the sign of the streamwise vorticity component (blue clockwise, red counter clockwise in front view) 


\section{Actuation taking into account geometrical constraints arising from hardware considerations}

A key feature of the AFLoNext project is that actuators have been developed in parallel to the aerodynamic numerical studies. This has allowed the sizing of the actuators to be considered. Because of the technology chosen for the Pulsed Jet Actuators (PJAs) and for the Synthetic Jet Actuators (SJAs) (see References [5] and [6]), size constraints were imposed on the actuators slot exit. To get enough peak velocity it was decided that the slot exit area should not exceed $5 \mathrm{~mm}^{2}$. Thus a notional slot size of $10 \mathrm{~mm}$ by $0.5 \mathrm{~mm}$ was selected. The spanwise spacing between actuators was set to $3 \mathrm{~mm}$ for PJAs and $1 \mathrm{~mm}$ for SJAs. Moreover, spacing between rows of actuators was also defined: $30 \mathrm{~mm}$ for PJAs and $50 \mathrm{~mm}$ for SJAs.

The most severe constraint was the slot width of $0.5 \mathrm{~mm}$, since it limited the blowing mass flow rate per unit spanwise length. This was taken into account by Dassault Aviation by considering a continuous blowing slot located at the leading edge separation line. The steady blowing was characterized by sonic jet exit velocities inclined at $20^{\circ}$ to the surface in the chordwise direction to favour momentum injection in the boundary layer. RANS computations were performed using the proprietary unstructured finite element flow solver Aether, developed by Dassault Aviation (see References [12] and [13]), using a zonal k-kl turbulence model for high lift configurations. As shown in Figure 13, the steady blowing through a $0.5 \mathrm{~mm}$ wide slot is still efficient at delaying the wing tip stall by $2^{\circ}$ of incidence and improves both lift and drag performance. It does as well as larger slots, but with a reduced mass flow injection. The corresponding flow topology is explained in Figure 14. Blowing through a $0.5 \mathrm{~mm}$ wide slot does not redirect the flow in the streamwise direction, but is enough to prevent the interaction of the tip vortex and the slat end vortex and to delay the breakdown of the latter vortex. Nevertheless the slat end vortex bursts at an incidence of $19^{\circ}$. To delay further the wing tip stall, blowing may be added in the wing tip root region in order to reinforce the slat end vortex.

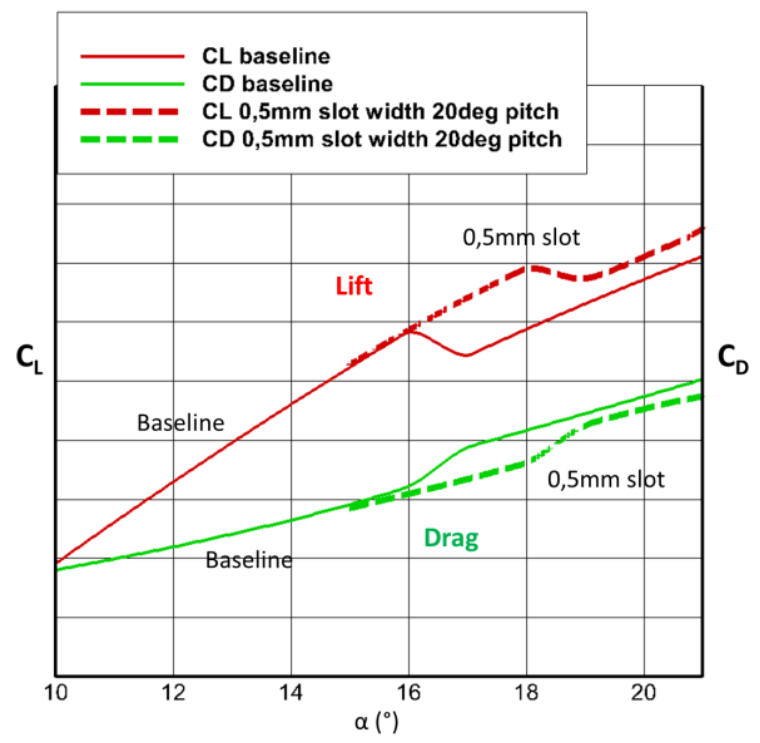

Figure 13: Lift and drag curve for the baseline and the configuration with steady sonic blowing $\left(\mathrm{U}_{\mathrm{jet}}=340 \mathrm{~m} / \mathrm{s}, \mathrm{C} \mu=0.026 \%\right)$ through a $0.5 \mathrm{~mm}$ wide slot at the leading edge separation line 

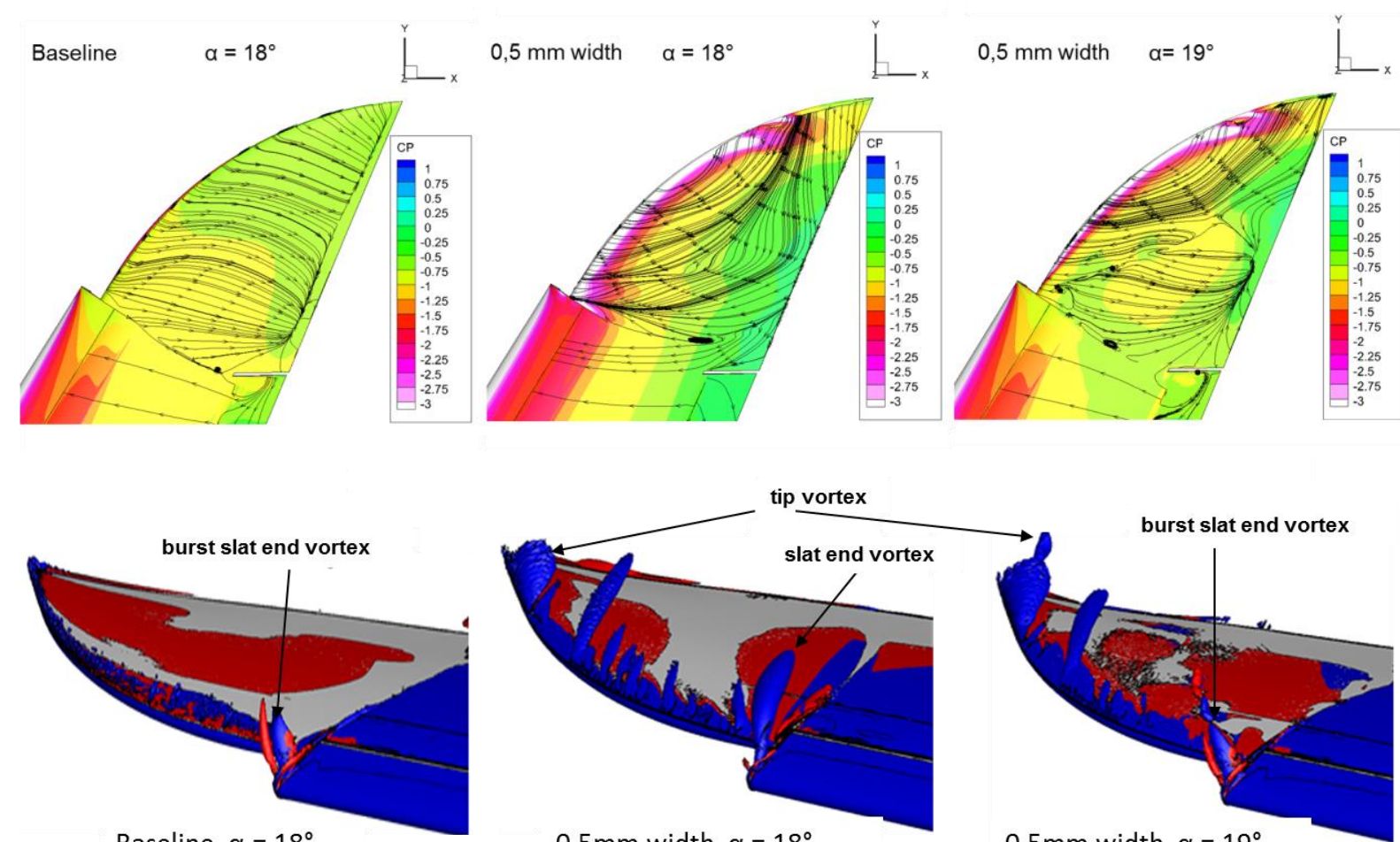

$0,5 \mathrm{~mm}$ width $\alpha=18^{\circ}$

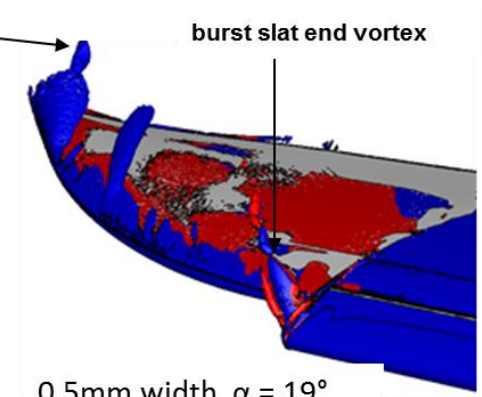

$0,5 \mathrm{~mm}$ width $\alpha=19^{\circ}$

Figure 14: Comparison of $C p$ distribution, skin friction lines and flow topology based on $Q$ criterion $(\mathrm{Q}=0.075)$ coloured by longitudinal vorticity (blue clockwise, red counter clockwise in front view) on the baseline at $18^{\circ} \mathrm{AoA}$ and on the $0.5 \mathrm{~mm}$ wide steady blowing slot at $18^{\circ}$ and $19^{\circ} \mathrm{AoA}\left(\mathrm{U}_{\mathrm{jet}}=340 \mathrm{~m} / \mathrm{s}\right.$, $\mathrm{C} \mu=0.026 \%$ ).

ONERA worked on the transposition of the previous AFC device at aircraft level in order to assess its effectiveness and efficiency on the XRF1 configuration, considering steady blowing with a reduced blowing velocity of $200 \mathrm{~m} / \mathrm{s}$ either through a continuous slot or a segmented one located at the leading edge separation line (see Fig. 16). The pitch angle relative to the surface was chosen as $30^{\circ}$, instead of $20^{\circ}$ previously, since the objective in a second step was to perform unsteady blowing and, based on DLR experience, this type of inclination angle favoured the interaction of the pulsed jet and the shear layer at the boundary of the flow separated zone. The slot width was $0.5 \mathrm{~mm}$ as previously. RANS and URANS computations were performed by ONERA using elsA structured software [11] with Spalart-Allmaras turbulence model. Figure 15 shows the performances of the two steady blowing concepts compared to the baseline. A significant improvement in L/D at aircraft level is associated with the two AFC devices, even if the blowing velocity is reduced in comparison to previous results. Moreover, the segmented slot is as effective as the continuous one, when the latter is effective, but its effectiveness extends across a broader range of incidence. This can be explained on the basis of flow topology comparisons, as illustrated in Figure 17. The segmented slot generates longitudinal vortices on the segment sides which help to stabilize the flow on the wing tip and delay the growth of the slat end vortex. The non-negligible benefit is also a reduction of the blowing mass flow by a factor 2 when using the segmented blowing slot. 


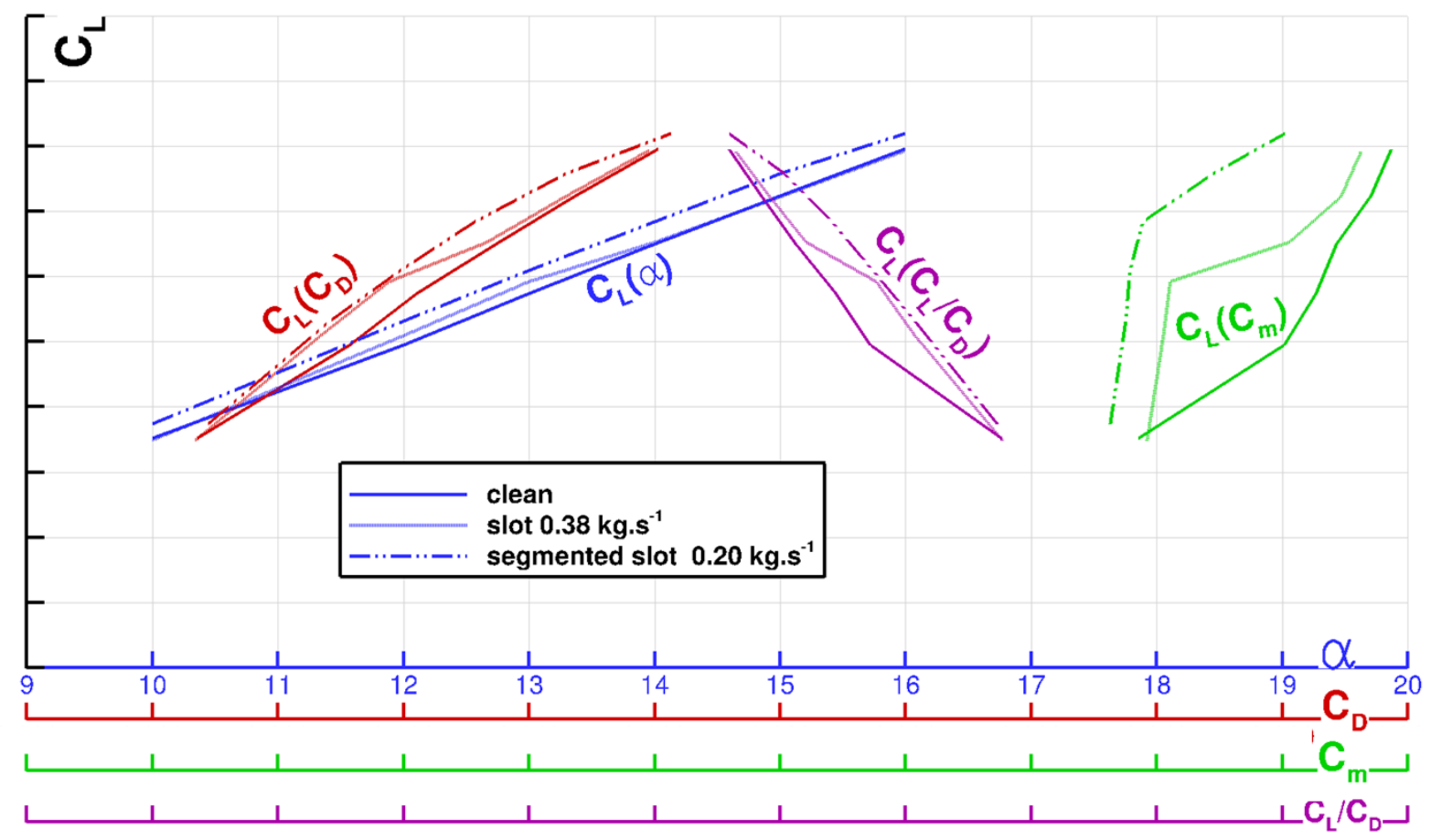

Figure 15: Lift, pitching moment, $C_{L} / C_{D}$ curves and drag polar for the baseline, the continuous slot $\left(U_{\text {jet }}=200 \mathrm{~m} / \mathrm{s}\right.$, massflow $\left.=0.38 \mathrm{~kg} / \mathrm{s}, C \mu=0.0072 \%\right)$ and the segmented slot $\left(U_{\text {jet }}=200 \mathrm{~m} / \mathrm{s}\right.$, massflow $=0.20 \mathrm{~kg} / \mathrm{s}, \mathrm{C} \mu=0.0038 \%$ ) on the full aircraft configuration
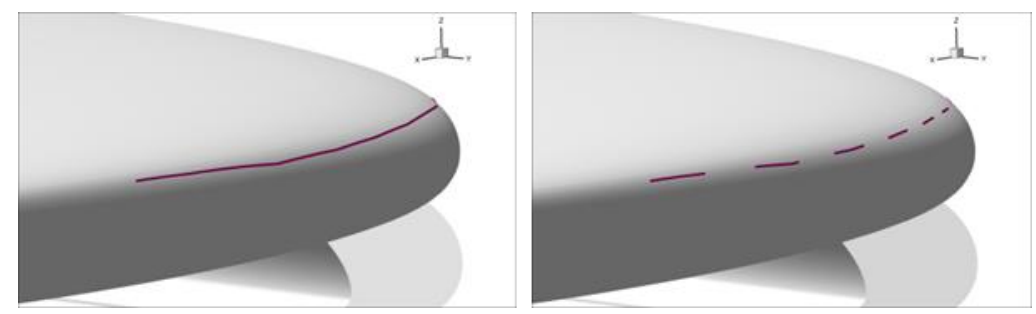

Figure 16: schematic view of continuous and segmented slots 


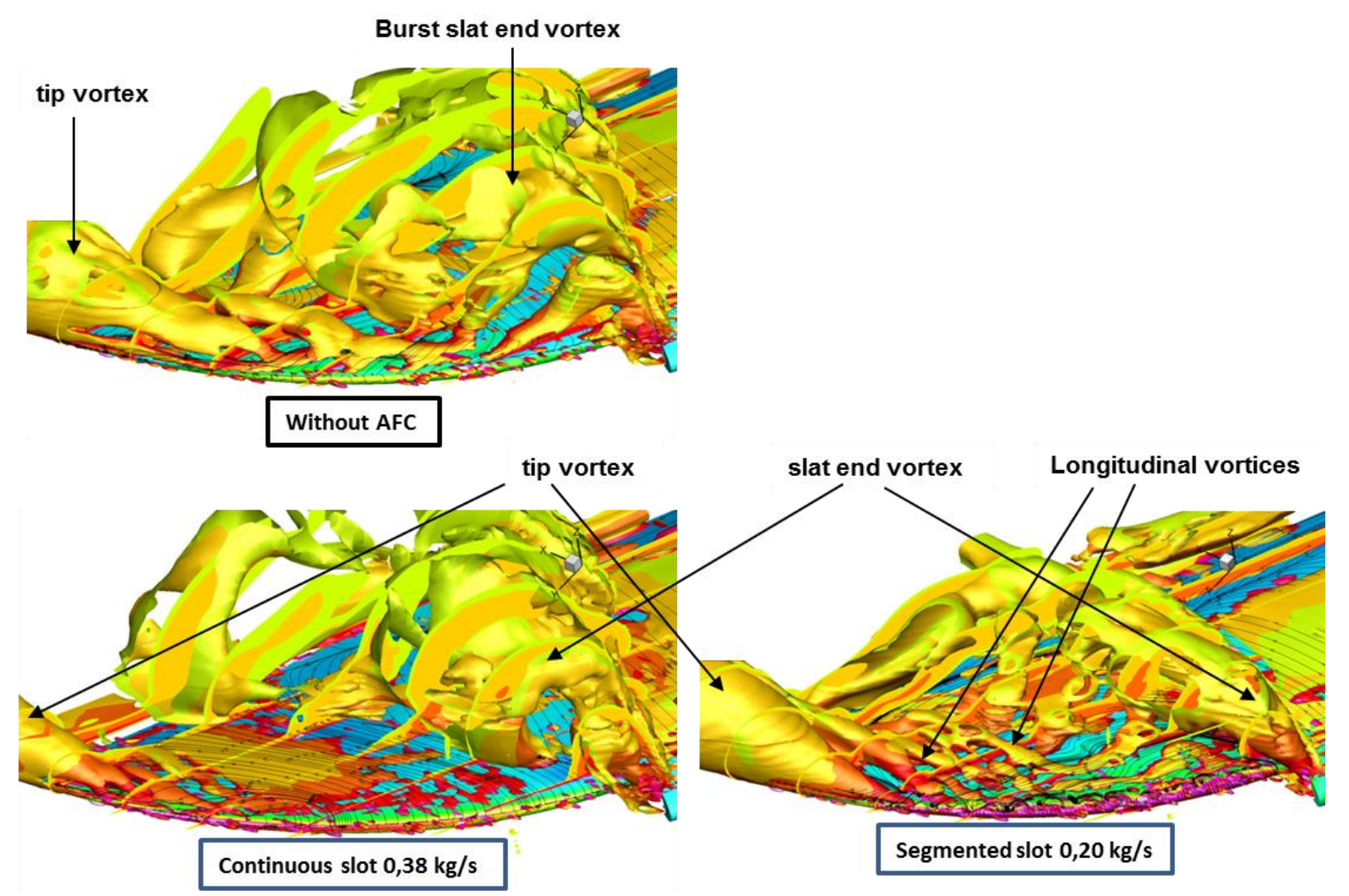

Figure 17: Comparison of flow topology (based on the Q-criterion coloured by vorticity) on the wing tip at $15^{\circ}$ AoA for the baseline configuration, the continuous blowing slot $\left(U_{\text {jet }}=200 \mathrm{~m} / \mathrm{s}\right.$, massflow $=0.38 \mathrm{~kg} / \mathrm{s}, \mathrm{C} \mu=0.0072 \%)$ and the segmented slot $\left(\mathrm{U}_{\mathrm{jet}}=200 \mathrm{~m} / \mathrm{s}\right.$, massflow $=0.20 \mathrm{~kg} / \mathrm{s}$, $\mathrm{C} \mu=0.0038 \%)$

In order to investigate the potential of pulsed jet actuation for the wing tip flow separation control, FOI and VZLU studied another set of segmented slots placed at the leading edge separation line, which corresponded to the integration of 248 actuator slots associated with PJAs. The slot size was $10 \mathrm{~mm}$ by $0.5 \mathrm{~mm}$ and the spacing between two slots was $3 \mathrm{~mm}$ (see Figure 18), in agreement with the device sizes chosen by the hardware developers. The blowing pitch angle to the surface was $30^{\circ}$ in the direction relative to the local streamlines. A refined mesh for DES computations (72 million nodes) was generated by VZLU and used by FOI both for RANS and DES computations. Such a refined mesh helps avoid the excessive dissipation of longitudinal vortices which are generated on the slot sides. RANS computations using the Edge solver [18] with Spalart-Allmaras turbulence model were performed by $\mathrm{FOI}$ on the baseline and on the steady blowing configurations, whereas IDDES simulations were run at $\mathrm{AoA} 18^{\circ}$ for the baseline, the steady blowing case and the pulsed jet case with a frequency of $50 \mathrm{~Hz}$. This frequency was chosen to make the unsteady simulation easier, since the influence of the frequency on the flow was not well known and extensive IDDES computations with various frequencies was too costly and not compatible with the project time frame. In all these computations peak blowing velocity was sonic. For the pulsed jet actuation the jet exit velocity profile with time was a square signal and the actuators were all in phase along the wing tip span.

The L/D improvement brought by AFC is presented in Figure 19. The wing tip stall is suppressed both with steady blowing and pulsed blowing from $12^{\circ}$ in AoA and delayed above $18^{\circ} \mathrm{AoA}$. For steady blowing the same effectiveness is obtained either with RANS or with IDDES, since most of the flow is 
attached on the wing tip (see Figure 20). A similar value of L/D is also obtained for the IDDES simulation of pulsed blowing at $18^{\circ}$ AoA. The corresponding flow is still attached on the wing tip and dominated by the effect of the slat end vortex. Although both types of blowing lead to similar improvements in L/D the corresponding flow topologies seem to be different. With steady blowing the skin friction lines start in the normal direction to the slots and then follow the streamwise direction. With pulsed blowing they are more influenced by the footprint of the slat end vortex.

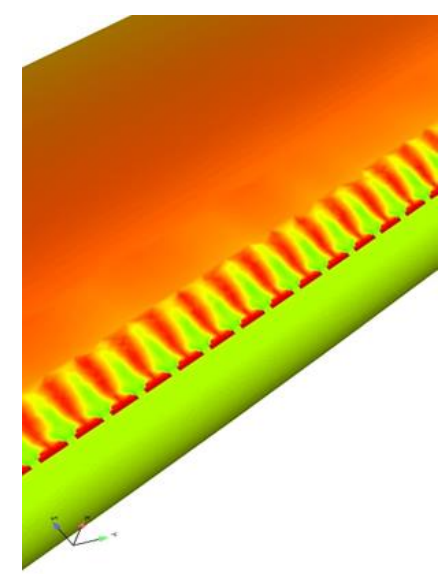

Figure 18: Configuration of actuator slots associated to the PJAs integrated on the leading edge separation line

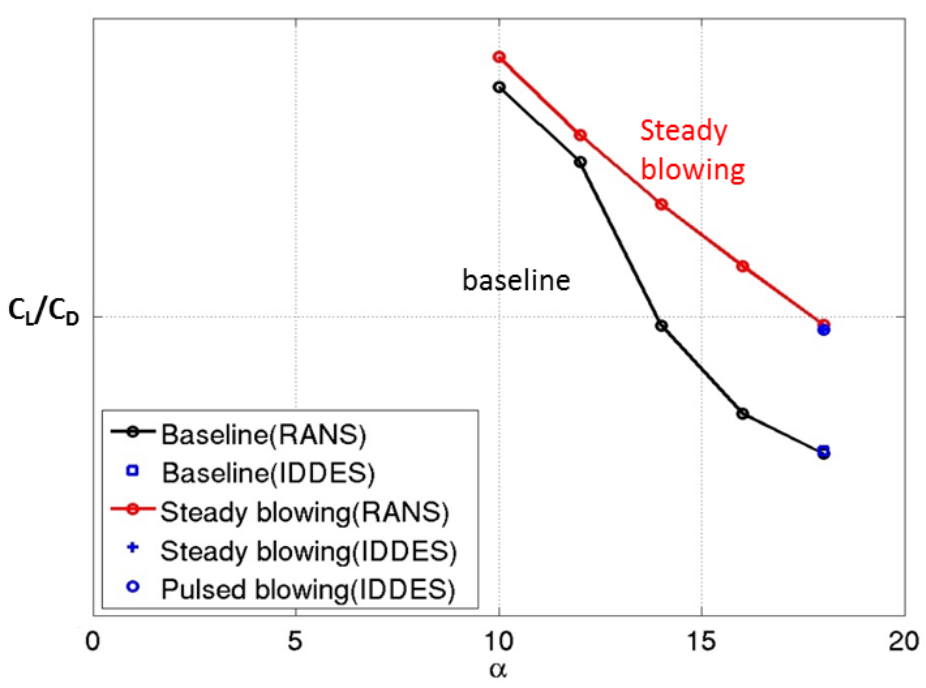

Figure 19: Comparison of $C_{L} / C_{D}$ on the outer wing for the baseline, steady blowing $\left(U_{j e t}=340 \mathrm{~m} / \mathrm{s}\right.$, massflow $=0.66 \mathrm{~kg} / \mathrm{s}, \mathrm{C} \mu=0.017 \%)$ and pulsed blowing configuration $\left(\mathrm{U}_{\text {jet peak }}=340 \mathrm{~m} / \mathrm{s}\right.$, massflow $=0.33 \mathrm{~kg} / \mathrm{s}, \mathrm{C} \mu=0.009 \%, \mathrm{f}=50 \mathrm{~Hz}$ ) 


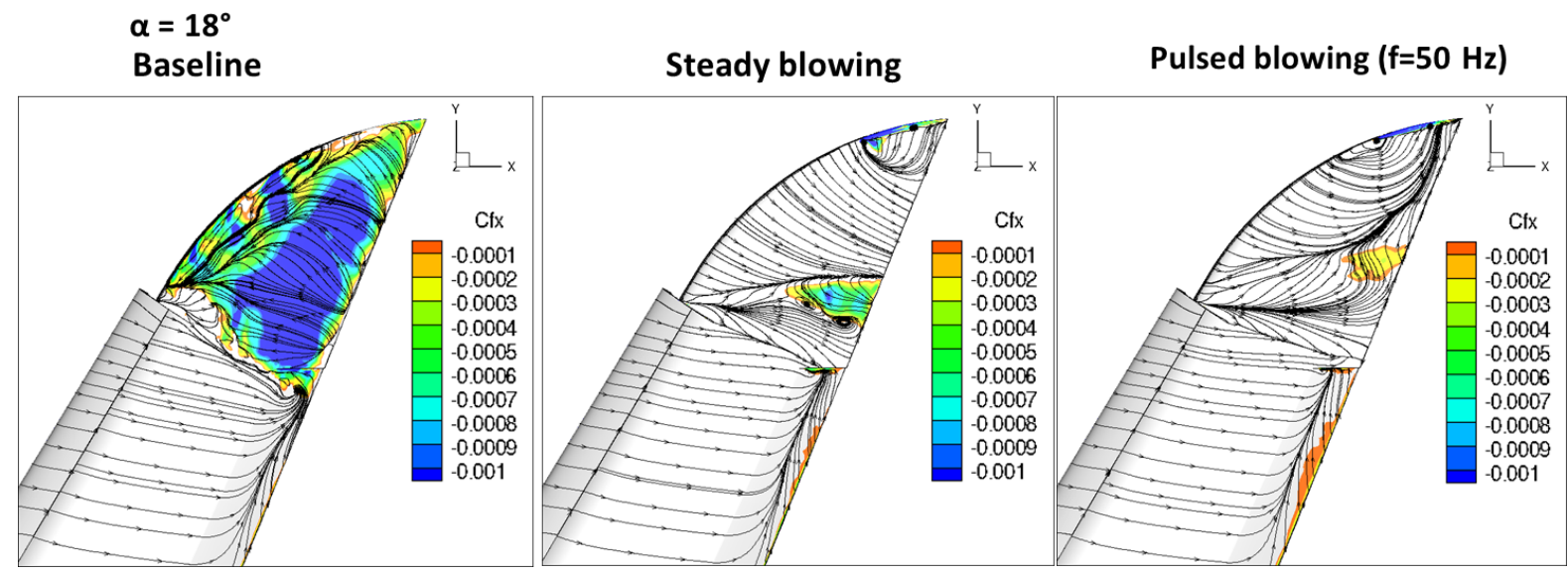

Figure 20: Comparison of $\mathrm{Cfx}$ and skin friction lines for the averaged IDDES results on the baseline, the steady blowing $\left(\mathrm{U}_{\mathrm{jet}}=340 \mathrm{~m} / \mathrm{s}\right.$, massflow $\left.=0.66 \mathrm{~kg} / \mathrm{s}, \mathrm{C} \mu=0.017 \%\right)$ and the pulsed blowing $\left(U_{\text {jet peak }}=340 \mathrm{~m} / \mathrm{s}\right.$, massflow $\left.=0.33 \mathrm{~kg} / \mathrm{s}, \mathrm{C} \mu=0.009 \%, \mathrm{f}=50 \mathrm{~Hz}\right)$ configurations at $18^{\circ} \mathrm{AoA}$ : coloured values represent negative values of $\mathrm{Cfx}$

\section{Synthesis of the AFC concepts}

A comparison and synthesis of the AFLoNext Partners' results was carried out in order to extract the general trends regarding AFC concepts on the outer wing at take-off. The figure of merit chosen was the percentage of improvement in L/D, since this is the relevant parameter at take-off, as a function of the blowing momentum coefficient $\mathrm{C} \mu$, which represents the energetic cost of AFC from a fluidic point of view (see Figure 21). It was chosen not to include the actuator energy cost in the figure of merit, since such actuators are still under development and their energy demands are not yet fully optimised. This figure of merit was computed for the condition just after the wing tip stall incidence of the baseline configuration, which varied from $14^{\circ}$ to $16^{\circ}$ depending on the partners' results. A second, complementary, measure used to quantify the effectiveness of AFC concepts was the shift in incidence of the wing tip stall realised through use of the AFC concept (see Figure 22).

Firstly the comparison focussed on steady blowing cases (Fig. 21). The best improvement obtained in $L / D$ is approximately $25 \%$. To get such an effectiveness, larger slots ( $4 \mathrm{~mm}$ wide) were needed on the upper surface, which corresponded to a $\mathrm{C} \mu$ value 3 times higher than the one associated to a $0.5 \mathrm{~mm}$ wide continuous slot located at the leading edge separation line. This clearly demonstrates that the leading edge separation line is a much more efficient location for AFC. The configuration with the segmented slot (corresponding to 248 actuator slots) allows further reduction in $\mathrm{C} \mu$ for an effectiveness which is even better, due to the stabilising effect of longitudinal vortices, which are created on the sides of the individual slots. Some further reduction in blowing momentum could be obtained by the reduction of blowing velocity from sonic to $200 \mathrm{~m} / \mathrm{s}$, as in ONERA calculations, but it would also lead to a reduction in effectiveness. The best improvement in incidence range was also obtained for the segmented slot configuration (248 actuator slots) and appeared to be greater than $5^{\circ}$. The exact value is not known, since no RANS computations were run by FOI above $18^{\circ}$ AoA.

The effect of the Synthetic Jet is also illustrated in Figure 21, where it is compared to the corresponding steady blowing configurations: these results concern the DLR calculations presented in Section 3. As observed previously, for a given peak velocity there is a reduction in effectiveness for the synthetic jet (15\% improvement in L/D) as compared to the steady blowing case (23\% 
improvement in L/D). But due to its sinusoidal behaviour, the blowing momentum of the synthetic jet is half that of the steady blowing. Decreasing the peak velocity of the SJA from $273 \mathrm{~m} / \mathrm{s}$ to $180 \mathrm{~m} / \mathrm{s}$ allows further reduction in $\mathrm{C} \mu$ for an improvement in L/D which remains close to the previous one (13\% improvement). In such a case reducing the input energy does not affect much the effectiveness of the flow control, since flow interaction mechanism and flow topology remain nearly unchanged (see Figure 10). The incidence range of improvement is of the order of $3^{\circ}$ for the synthetic jets located on the upper surface.

Figure 21 also shows the effect of the pulsed jet compared to steady blowing. The FOI results of Section 4 have shown that similar effectiveness could be obtained between pulsed jets and the corresponding steady blowing case, but with a $\mathrm{C} \mu$ value which is reduced by a factor of 2 . This PJA configuration turns out to be the most effective and efficient of the numerically investigated AFC concepts with $26 \%$ improvement in L/D and a $C \mu$ value per wing lower than 0.0001 . As for the associated steady blowing case, the incidence range of improvement is greater than $5^{\circ}$. On the basis of other URANS computations of pulsed jet actuators performed by VZLU on the same configuration, it appeared that considering neighbouring actuators with a $180^{\circ}$ phase shift to reduce the acoustic contribution of actuators would lead to a $25 \%$ reduction of the effectiveness as compared to in phase actuators.

The massflow of all the compared AFC concepts except the steady blowing at $10 \%$ chord was within the limits of the bleed air requirement.
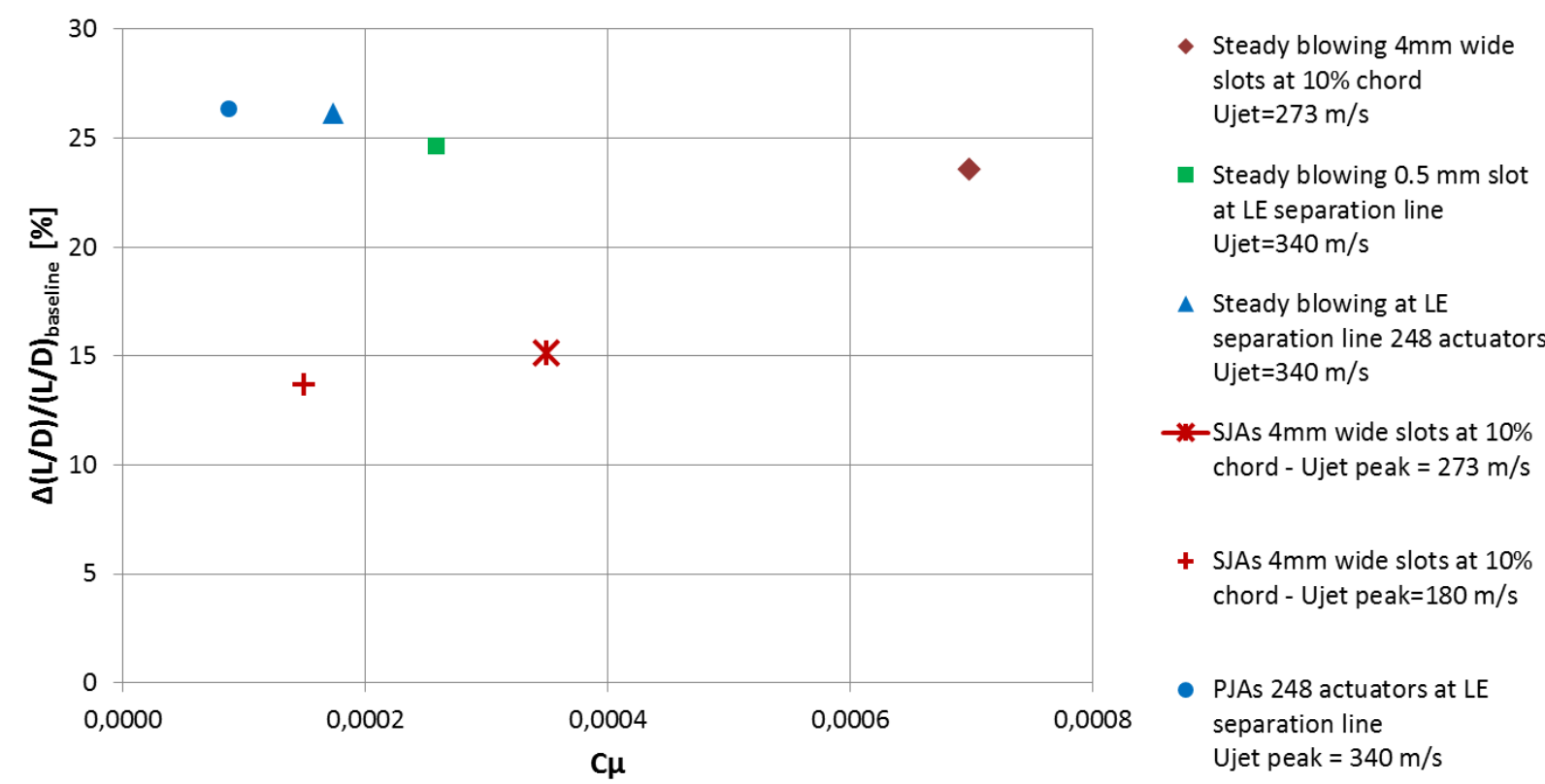

Figure 21: Comparison of the most effective AFC concepts with regard to the improvement in L/D as a function of $\mathrm{C} \mu$ related to one wing 


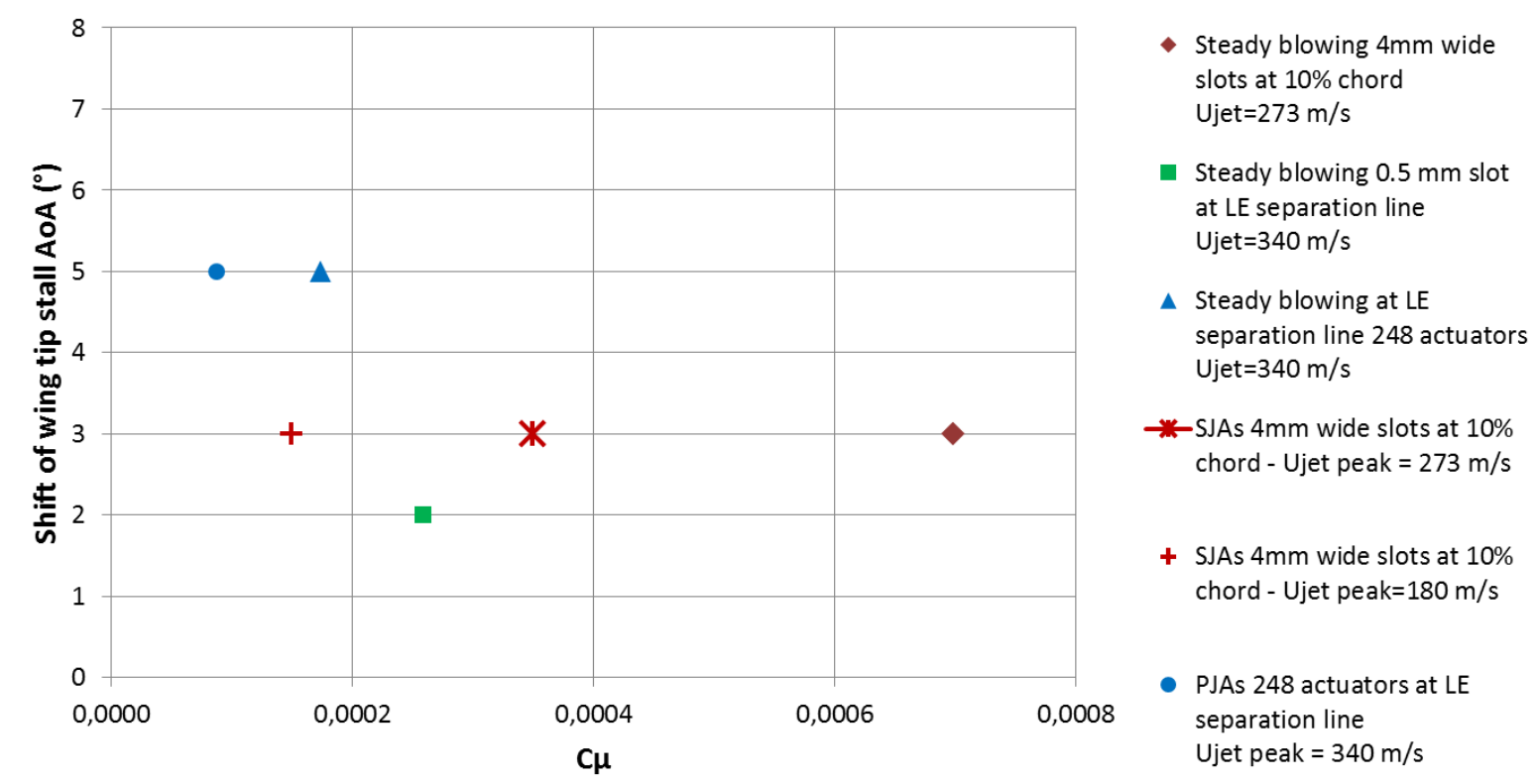

Figure 22: Comparison of the most effective AFC concepts with regard to the shift of wing tip stall incidence as a function of $\mathrm{C} \mu$ related to one wing

\section{Conclusion}

The potential of AFC to delay flow separation in the outer wing region has been investigated in the AFLoNext project using CFD. The aim was to improve the L/D at take-off. As expected, the best AFC locations were found:

- Either close to the leading edge separation line to prevent vortex interaction and development

- Or at the wing tip root to strengthen the slat end vortex

Differences in the flow mechanisms between steady blowing and synthetic jets have been underlined: the former injects momentum inside the boundary layer, whereas the latter generate spanwise rows of stabilising vortices.

Taking into account sizing constraints arising from hardware development the project has shown the benefit of segmented thin slots, where longitudinal vortices help stabilising the flow. On such a configuration pulsed jets were found to be as effective as steady blowing, but using half the blowing mass flow. Moreover, updated requirements regarding peak velocities were also derived to specify the AFC hardware developed within the project.

An overall synthesis was performed in order to extract general trends about best AFC location and actuation type. The most efficient AFC approach was found to be the pulsed jet blowing through segmented slots located at the leading edge separation line. It gave an overall improvement of $26 \%$ in L/D at take-off on the outer wing geometry at the cost of a blowing massflow of $.33 \mathrm{~kg} / \mathrm{s}$ per wing, which is within the limits of the available bleed air. 
In further studies this AFC approach should be compared on the XRF1 wing tip configuration to two other types of actuation:

- sweeping jets or switching jets based on fluidic oscillators located at the leading edge separation line, which were not investigated in the present study, as it is known from other research that they could work and may allow further reduction in $\mathrm{C} \mu$ as compared to the PJAs.

- steady suction or Suction and Oscillatory Blowing actuators placed in the wing tip root region to stabilize the slat end vortex.

The present study was performed on a specific geometry of wing tip extension with a curved leading edge, but similar conclusions could be extended to configurations with a winglet, provided that flow separation occurs at the foot of the winglet. Specific configurations where the flow separates on the winglet itself would need further investigations.

\section{Acknowledgments}

The work described in this paper and the research leading to these results have received funding from the European Community's Seventh Framework Programme FP7/2007-2013, under grant agreement $n^{\circ} 604013$, AFLONEXT project.

\section{References}

[1] Getting to grips with - aircraft performance, Flight Operations Support \& Line Assistance, Customer Services Airbus, and at http://www.smartcockpit.com/aircraftressources/Getting_to_Grips_With_Aircraft_Performance.html available, October 2013

[2] Flaig, A. and Hilbig, R., "High-lift design for large civil aircraft," in High-Lift System Aerodynamics, AGARD CP 515, pp. 31.1-31.12, 1993.

[3] Greenblatt, D., and Wygnanski, I, "The Control of Flow Separation by Periodic Excitation," Progress in Aerospace Sciences, Vol. 36, No. 7, 2000, pp. 487-545.

[4] Joslin, R.D., Miller, D.N., "Fundamentals and Applications of Modern Active Flow Control", Progress in Astronautics and Aeronautics, Vol. 231, ISBN 978-1-56347-983-0, AIAA, 2009.

[5] Weigel, P., Schüller, M., ter Meer, T., Bardet, M., "Design of a Synthetic Jet Actuator for Separation Control", Proceedings of CEAS2017 conference.

[6] Schlösser, P., "Design of a Pulsed Jet Actuator for Separation Control”, Proceedings of CEAS2017 conference.

[7] Ciobaca, V., Wild, J., "Active Flow Control for an Outer Wing Model of a Take-off Transport Aircraft Configuration - A Numerical Study," AIAA Paper 2014-2403, 2014.

[8] Ciobaca, V., Wild, J., Bauer, M., Grund, T., Huehne, P., Scholz, P.,Stefes, B., "Wind Tunnel Experiments with Active Flow Control for an Outer Wing Model," AIAA Paper 2015-2728, 2015. 
[9] Bauer, M., Grund, T.,Nitsche, W. - "Experiments on Active Drag Reduction on a Complex Outer Wing Model” - AIAA Journal Vol. 53, N 7, July 2015, pp. 1774-1783.

[10]Detinis, I., Steinbuch, M., Segal, S., Lagutin, M., Seifert, A., Wasserman, M., Levi, Y. - "Active Flow Control Implementation for Mitigating Outer Wing Flow Separation" - 57th Israel Annual Conference on Aerospace Sciences, 15-16 March 2017.

[11] Cambier, L., Heib, S., Plot, S., The Onera elsA CFD Software: Input from Research and Feedback from Industry, Mechanics \& Industry, Num. 3, Vol. 14, Apr. 2013.

[12] Chalot, F., Mallet, M., Ravachol, M., "A comprehensive finite elements Navier-Stokes solver for low and high speed aircraft design", Reno, AIAA94-0814, January 1994.

[13] Chalot, F., "Industrial Aerodynamics", Encyclopedia of Computational Mechanics, Volume 3, Wiley (2004).

[14] Gerhold, T., "Overview of the Hybrid RANS Code TAU," MEGAFLOW - Numerical Flow Simulation for Aircraft Design, Vol. 89 of Notes on Numerical Fluid Mechanics and Multidisciplinary Design, Springer Verlag, 2005, pp. 81-92.

[15] Ciobaca, V., and Wild, J. "An Overview of Recent DLR Contributions on Active Flow-Separation Control Studies for High-Lift Configurations," AerospaceLab Journal, Issue 6 "Flow Control: the Renewal of Aerodynamics?", June 2013.

[16] Ciobaca, V., Kühn, T., Rudnik, R., Bauer, M., Gölling, B. and Breitenstein, W., "Active Flow Separation Control on a High-Lift Wing-Body Configuration," Journal of Aircraft, Vol.50, No.1, 2013, pp.56-72.

[17] Ciobaca,V., Haucke, F., Rudnik, R. and Nitsche, W. „Active Flow Control on a High-Lift Airfoil: URANS Simulations and Comparison with Time-Accurate Measurements," AIAA Paper AIAA 20132795, 2013.

[18] Eliasson, P., "Edge, a Navier-Stokes Solver for Unstructured Grids", Proceedings of Finite Volumes applications III, ISBN 1-9039-9634-1, pp. 527-534, 2002. 